\title{
Multi Objective Optimization for UAV Navigation Equipment Test Table Design
}

\author{
V.M. Sineglazov \\ Aviation Computer-Integrated Complexes Department \\ National Aviation University, Kyiv, Ukraine \\ svm@nau.edu.ua
}

\begin{abstract}
Real world test equipment design optimization problems are a class of challenging multiobjective optimization problems, which may contain two levels of optimization tasks. In these problems, the optimal solutions to the lower level problems become possible feasible candidates to the upper level problem. A bilevel optimization is used for the navigation equipment test table design. The six lower level optimizations are driven by a top system level optimization by approach based on hybrid bilevel evolutionary algorithm using quadratic approximations.
\end{abstract}

Keywords- multiobjective optimization; bilevel optimization; simulation table; evolutionary algorithm.

\section{INTRODUCTION}

The complexity of modern highly integrated designs has sparked increasing interest in multiobjective optimization. Navigation equipment test table as means of providing technical testing navigation equipment in conditions close to the real flight [1]. Navigation equipment test table must ensure tests on the parameters close to real, namely the angular positions, overload, angular velocity, acceleration, etc at the same time conforming the requirements of the very device under the test. Moreover it must ensure required reliability and credibility performance.

A dozen performances of dynamic platform, gears, electric drives, electric drive control subsystem, data acquisition subsystem, power supply sub-system, signal processors during design process must be optimal to ensure all test conditions with the reasonable test table cost (Fig. 1).

This design approach provides the results to a global design problem which improves the lower level objective functions while at the same time producing the highest possible improvement at the system upper level.

Bilevel optimization as a branch of optimization, which contains a nested optimization problem within the constraints of the outer optimization problem can be described as a methodology for the design of systems, where the optimization of sever-al lower level objective functions must be considered and they are constraints at the same time to the upper system level problem.

To address complexity of the navigation equipment test table design task hierarchical decomposition approach may

\author{
S.O. Dolgorukov \\ Aviation Computer-Integrated Complexes Department \\ National Aviation University, Kyiv, Ukraine \\ sdolgorukov@nau.edu.ua
}

be used. A hierarchic system is defined as one in which a subsystem exchanges data directly with the system only but not with any other subsystem. Such data exchange occurs in analysis of structures by substructuring. A concept to exploit this in structural optimization was formulated in Schmit and Ramanathan [2] and generalized in Sobieszczanski-Sobieski [3] and [4]. It was then shown in the latter how the hierarchic decomposition derives from the Bellman's optimality criterion of the dynamic programming. The concept was also contributed to by Kirsch, [e.g., Kirsch, 5]. It was demonstrated in several applications, including multidisciplinary ones, e.g., Wrenn and Dovi [6] and Beltracchi [7].

But the hierarchical optimization structure may introduce difficulties such as non-convexity and disconnectedness even for simpler instances of bi-level optimization like bilevel linear programming problems. Bilevel linear programming is known to be strongly NPhard [8], and it has been proven that merely evaluating a solution for optimality is also a NP-hard task [9]. This gives us an idea about the kind of challenges offered by bilevel problems with complex (non-linear, non-convex, discontinuous) objective and constraint functions.

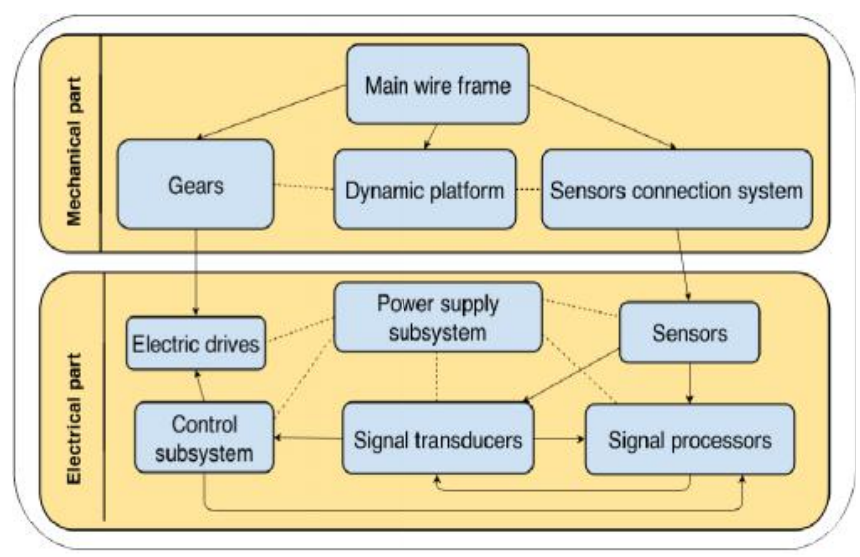

Fig. 1. Navigation equipment test table.

In the field of classical optimization, a number of studies have been conducted on bilevel programming [10], [11]. Approximate solution techniques are commonly employed to handle bilevel problems with simplifying assumptions 
like smoothness, linearity or convexity. Some of the classical approaches commonly used to handle bilevel problems in-clude the Karush-Kuhn-Tucker approach [12], Branch-and-bound techniques [13], and the use of penalty functions [14]. Most of these solution methodologies are rendered inapplicable, as soon as the bilevel optimization problem becomes complex. Heuristic procedures such as evolutionary algorithms have also been developed for handling bi-level problems with higher levels of complexity [15]. Most of the existing evolutionary procedures often involve enormous computational expense, which limits their utility to solving bilevel optimization problems with smaller number of variables.

There are a number of practical problems which are bilevel in nature. They are often encountered in transportation (network design, optimal pricing) [16], [17], economics (Stackelberg games, principal agent problem, taxation, policy decisions) [18]-[20], management (network facility location, coordination of multi-divisional firms) [21], [22], engineering (optimal design, optimal chemical equilibria) [23], [24]. Complex practical problems are usually modified into a simpler single level optimization task, which is solved to satisfy instead of an optimal solution. For the complex bilevel problems, classical methods fail due to real world difficulties like non-linearity, discreteness, non-differentiability, non-convexity etc. Evolutionary methods are not very useful either because of their enormous computational expense. Under such a scenario, a hybrid strategy could be solution. Acknowledging the drawbacks associated with the two approaches, we propose a hybrid strategy that utilizes principles from classical optimization within an evolutionary algorithm to quickly approach a bilevel optimum. The proposed method for multi objective optimization for navigation equipment test table design is a bilevel evolutionary algorithm based on quadratic approximations of the lower level optimal variables as a function of upper level variables.

\section{TEST TABLE MULTIOBJECTIVE OPTIMIZATION}

Consider the navigation equipment test table upper level design problem formulated in terms of a design variable vector $x_{u}[H, P, N, G, Q, L, E, R]$, where

$H$ is the size of the mounting for the equipment under test;

$P$ is the mass of the equipment under the test;

$N$ is number of test table rotation axes;

$G$ is limits of the angular velocity;

$Q$ is stability of the angular velocity;

$L$ is test results measurement accuracy;

$E$ is the accuracy of the setting the test results digital information;

$R$ is reliability.

There are two types of variables is the upper level variables $x_{u} \in X_{U} \subset R^{n}$, and the lower level variables $x_{1} \in X_{L}$ $\subset R^{m}$.
Lower level objective function $f$ is comprised of six subproblems $f_{1}, f_{2}, f_{3}, f_{4}, f_{5}, f_{6}$. The design variables and constraints for each of these lower level problems are denoted by $x_{11}, x_{12}, x_{13}, x_{14}, x_{15}, x_{16}$, and $g_{x 1}, g_{x 2}, g_{x 3}, g_{x 4}, g_{x 5}$ and $g_{x 6}$, respectively (Table I).

TABLE I. DESIGN VARIABLE DEFINITIONS

Variable Definition

\begin{tabular}{|c|c|}
\hline & $X_{11}$ - dynamic platform \\
\hline$M$ & Test equipment load capacity \\
\hline$m$ & Mass of platform \\
\hline \multirow[t]{2}{*}{$D$} & Dimensions of platform \\
\hline & $x_{12}-$ gears \\
\hline$r$ & Dependability \\
\hline$d$ & Types of sizes \\
\hline$a$ & The degree of accuracy \\
\hline$s$ & The gear ratio \\
\hline$T$ & Output torque \\
\hline \multirow[t]{2}{*}{$B$} & Mechanical backlash \\
\hline & $x_{13}$ - electric drives \\
\hline$b$ & The mechanical stiffness of the drive \\
\hline$h$ & Weight and dimensions \\
\hline$l$ & Reliability \\
\hline \multirow[t]{2}{*}{$S$} & Response performance of the drive \\
\hline & $\mathbf{x}_{14}$ - electric drive control subsystem \\
\hline$u$ & Performance of control equipment \\
\hline$j$ & Smoothness of the drive motion control \\
\hline \multirow[t]{2}{*}{$g$} & Control accuracy \\
\hline & $x_{15}$ - data acquisition subsystem \\
\hline$A$ & Accuracy \\
\hline$n$ & Sampling frequency \\
\hline \multirow[t]{2}{*}{$i$} & Noise immunity \\
\hline & $x_{16}-$ power supply subsystem \\
\hline$K$ & Efficiency \\
\hline$o$ & Fault tolerance \\
\hline
\end{tabular}

The lower level multi-objective problem is solved with respect to the lower level variables, while the upper level variables act as parameters to the optimization problem. The follower is interested in optimizing its own six objectives and making its own decision for the test table upper level vector. In this case the leader wants to solve such a problem where the follower has sufficient decision making power, so then it needs to have a complete knowledge of the follower's decision structure. The decision structure of the follower may is represented in the form of a value function. For the test table upper-level objective function $F: \mathrm{R}^{\mathrm{n}} \times \mathrm{R}^{\mathrm{m}}$ $\rightarrow \mathrm{R}^{\mathrm{p}}$ and lower-level objective function $f: \mathrm{R}^{\mathrm{n}} \times \mathrm{R}^{\mathrm{m}} \rightarrow \mathrm{R}^{\mathrm{q}}$

$$
\text { minimize } F\left(x_{u}, x_{1}\right)=\left(F_{1}\left(x_{u}, x_{1}\right), . ., F_{p}\left(x_{u}, x_{1}\right)\right)
$$




$$
\begin{aligned}
& \text { subject to } \\
& \begin{array}{l}
x_{l} \in \operatorname{argmin}_{x l}\left\{V \left(f\left(x_{u}, x_{1}\right)=\left(f_{1}\left(x_{u}, x_{1}\right), \ldots, f_{q}\left(x_{u}, x_{1}\right) ; \omega\right):\right.\right. \\
\left.g_{j}\left(x_{u}, x_{1}\right) \leq 0, j=1, . ., J\right\} \\
G_{k}\left(x_{u}, x_{1}\right) \leq 0, k=1, \ldots, K,
\end{array}
\end{aligned}
$$

where $V$ denotes the follower's value function, and $\omega$ is the parameter vector of the assumed value function form.

We assume that the leader has a complete knowledge of the follower's value function. Based on this information, we solve the bilevel problem to identify the upper level Paretofrontier. Once the upper level Pareto-frontier is available to the leader, it becomes a multi-criteria decision making problem for the leader.

An evolutionary algorithm [24] for solving bilevel problems where the upper level has multiple objectives and the lower level decisions are modeled using a value function is known as multi-objective bilevel evolutionary algorithm based on quadratic approximations (m-BLEAQ). The proposed approach is based on estimation of unknown lower level decisions using quadratic approximations, when lower level decisions corresponding to a few upper level vectors are known. The approximation helps in reducing the number of lower level optimization calls that leads to computational savings.

A steady state evolutionary algorithm 1 for global optimization is used at the lower level to find the optimum. The fitness assignment at the lower level is performed based on lower level function value and constraints. The upper level vector for which lower level optimization is beingperformed is kept fixed during the optimization run.

Step 1. Randomly initialize a lower level population of size $N$. Assign fitness to the members based on lower level objective functions and constraints.

Step 2. Randomly choose 6 members from the population, and perform a tournament selection.

This gives 3 parents for crossover.

Step 3. Create 2 offsprings from the parents using genetic operators on the lower level variables only.

Step 4. Randomly choose 2 members from the population, and pool them with 2 offsprings. The 2 best members from the pool replace the chosen members from the population.

Step 5. Perform a termination check. Proceed to next generation (Step 2), if the termination criteria is not satisfied, otherwise proceed to the next step.

Step 6. The best obtained lower level member is paired with the corresponding upper level member in the upper level population.

Fitness assignment for feasible upper level member is performed based on their non-domination rank and crowding distance [25]. For a given upper level member $x$, if the non-domination rank is given as $N_{R}(x)$ and crowding distance within its frontier is given as $C_{D}(x)$, then the fitness for the member is calculated as follows:

$$
F_{u}(x)=1 /\left(N_{R}(x)+e^{-C D(x)}\right) .
$$

Fitness for an infeasible upper level member is computed by subtracting the sum of upper level constraint violations from the fitness value of the worst feasible member.

The fitness during lower level optimization is given by lower level function values for the feasible members. For the infeasible lower level members, we subtract the sum of lower level constraint violations from the fitness value of the worst feasible member at that level.

The crossover operator is similar to the PCX operator proposed in [26] with slight modifications. The operator requires 3 parents to create an offspring that are selected using tournament selection.

$$
c=x^{(p)}+\omega_{\alpha} d+\omega_{\beta}\left(p^{(2)}-p^{(1)}\right) / 2
$$

where $x^{(p)}$ is the index parent; $d=x^{(p)}-\mathrm{g}$, where $g$ is the mean of $\mu$ parents; $p^{(1)}$ and $p^{(2)}$ are the other two parents; $\omega_{\alpha}=0.1$ and $\omega_{\beta}=\operatorname{dim}\left(x^{(p)}\right) /\left\|x^{(p)}-g\right\|_{1}$ are the two parameters.

At any generation of the m-BLEAQ algorithm, we attempt to maintain at least $N / 2$ tag 1 members. These are the upper level members for which the lower level optimal solutions are accurately known. We utilize these members to compute the lower level optimal solutions of the new upper level members. When multiple lower and upper level variables are present, we utilize all the upper level variables to construct the quadratic approximation for each lower level variable. Therefore, the number of quadratic approximations are as many as the number of lower level variables, and each lower level variable is a function of all the upper level variables. We choose the closest upper level members for quadratic approximation around the point for which we intend to estimate the lower level decision. Such an approximation is expected to provide a reliable local estimate. It is necessary to utilize at least $1 / 2$ $\left[\left(\operatorname{dim}\left(x_{u}\right)+1\right)\left(\operatorname{dim}\left(x_{u}\right)+2\right)\right]+\operatorname{dim}\left(x_{u}\right)$ upper level points for constructing the approximation.

The upper level population is updated by choosing 2 random members from the population. The members are pooled with 2 offsprings generated through genetic operations, and the best members from the pool are chosen to replace the selected population members.

At the upper level we terminate the algorithm based on maximum upper level function evaluations $\left(T_{\max }\right)$. We use an improvement based termination at the lower level such that if the improvement in the lower level function value is less than $1 \mathrm{e}-5$ for 100 consecutive generations then we terminate the optimization.

Comparison of m-BLEAQ [24] and H-BLEMO [27] performance by Inverted Generalization Distance (IGD) [28] metric prove that H-BLEMO is capable of handling multiple objectives at both levels, but in the current formulation of simulation table design problem the lower level is represented by a value function, which means that 
H-BLEMO is handling a single objective problem at the lower level and a multi-objective problem at the upper level. On the other hand m-BLEAQ cannot directly handle multiple objectives at both levels. However, with multiple objectives at upper level and single objective at lower level m-BLEAQ is able to achieve much lower IGD values as compared to H-BLEMO for the same number of upper level function evaluations and much fewer lower level function evaluations.

\section{CONCLUSIONS}

Hybrid multi objective optimization approach for navigation equipment test table design based on evolutionary algorithm for development is a rational methodology by which the multilevel design problem could be partitioned into a number of up- and low level problems.

Further implementation in development of the software algorithm could be made to assess multiple design alternatives of navigation equipment test table and summarize their results for fast comparison. Such approach enables engineers to quickly understand the benefits of different design technical solutions to gain greater insight into how individual components are performing.

\section{REFERENCES}

[1] V.M.Sineglazov, S.O.Dolgorukov:HardWare-In-The-Loop simulation table for UAV navigation complexes testing. APUAVD, 2013 IEEE 2nd International Conference , pp. 141-145, 2013.

[2] Schmit, L. A.; and Ramanathan, R. K.: Multilevel Approach to Minimum Weight Design including Buckling Constraints. AIAA J., vol. 16, pp. 97-104, 1978.

[3] Sobieszczanski-Sobieski, J.: Optimization by Decomposition: A Step from Hierarchic to Non-Hierarchic Systems. Presented at the Second NASA/Air Force Symposium on Recent Advances in Multidisciplinary Analysis and Optimization, Hampton, VA, September 28-30, 1988. NASA TM101494. NASA CP-3031, Part 1, 1989.

[4] Sobieszczanski-Sobieski, J.: Sensitivity of Complex, Internally Coupled Systems. AIAA Journal, vol. 28, No. 1, 1990, pp. 153-160.

[5] Kirsch, U. Optimum Structural Design, McGraw-Hill, 1981.

[6] Wrenn, G. A.; and Dovi, A. R.: Multilevel Decomposition Approach to the Preliminary Sizing of a Transport Aircraft Wing. AIAA Paper 87-0714-CP, April 1987.

[7] Beltracchi, T. J.: A Decomposition Approach to Solving the Allup Trajectory Optimization Problem, AIAA Paper 90-0469 presented at the AIAA Aerospace Sciences Meeting, Reno, NV, 1990, also in AIAA J. of Guidance Control and Dynamics, Nov. 1991.

[8] P. Hansen, B. Jaumard, and G. Savard. New branch-and-bound rules for linear bilevel programming. SIAM Journal on Scientific and Statistical Computing, 13(5):1194-1217, 1992.

[9] L. N. Vicente, G. Savard, and J. J. Judice. Descent approaches for quadratic bilevel programming. Journal of Optimization Theory and Applications, 81(1):379-399, 1994.

[10] B. Colson, P. Marcotte, and G. Savard. An overview of bilevel optimization. Annals of Operational Research, 153:235-256, 2007.

[11] L. N. Vicente and P. H. Calamai. Bilevel and multilevel programming: A bibliography review. Journal of Global Optimization, 5(3):291-306, 2004

[12] L. Bianco, M. Caramia, and S. Giordani. A bilevel flow model for hazmat transportation network design. Transportation Research Part C: Emerging technologies, 17(2):175-196, 2009.

[13] J.F. Bard and J. Falk. An explicit solution to the multi-level programming problem. Computers and Operations Research, 9:77$100,1982$.
[14] E. Aiyoshi and K. Shimizu. Hierarchical decentralized systems and its new solution by a barrier method. IEEE Transactions on Systems, Man, and Cybernetics, 11:444-449, 1981.

[15] G. Wang, Z. Wan, X. Wang, and Y. Lv. Genetic algorithm based on simplex method for solving linear-quadratic bilevel programming problem. Comput Math Appl, 56(10):2550-2555, 2008.

[16] Isabelle Constantin and Michael Florian. Optimizing frequencies in a transit network: a nonlinear bi-level programming approach. International Transactions in Operational Research, 2(2):149-164, 1995.

[17] Luce Brotcorne, Martine Labbe, Patrice Marcotte, and Gilles Savard. A bilevel model for toll optimization on a multicommodity transportation network. Transportation Science, 35(4):345-358, 2001.

[18] F. J. Wang and J. Periaux. Multi-point optimization using gas and Nash/Stackelberg games for high lift multi-airfoil design in aerodynamics. In Proceedings of the 2001 Congress on Evolutionary Computation (CEC-2001), pp. 552-559, 2001.

[19] Mark Cecchini, Joseph Ecker, Michael Kupferschmid, and Robert Leitch. Solving nonlinear principalagent problems using bilevel programming. European Journal of Operational Research, 230(2), 364-373, 2013.

[20] A. Sinha, P. Malo, A. Frantsev, and K. Deb. Multi-objective stackelberg game between a regulating authority and a mining company: A case study in environmental economics. In 2013 IEEE Congress on Evolutionary Computation (CEC-2013). IEEE Press, 2013.

[21] Hande Kkaydin, Necati Aras, and I. Kuban Altnel. Competitive facility location problem with attractiveness adjustment of the follower: A bilevel programming model and its solution. European Journal of Operational Research, 208(3):206-220, 2011.

[22] J. F. Bard. Coordination of multi-divisional firm through two levels of management. Omega, 11(5):457-465, 1983.

[23] C. Kirjner-Neto, E. Polak, and A.Der Kiureghian. An outer approximations approach to reliabilitybased optimal design of structures. Journal of Optimization Theory and Applications, 98(1):116, 1998.

[24] J.F. Bard. Practical Bilevel Optimization: Algorithms and Applications. The Netherlands: Kluwer, 1998.

[25] A. Sinha, P. Malo, and K. Deb. Towards understanding bilevel multiobjective optimization with deterministic lower level decisions. In Proceedings of the Eighth International Conference on Evolutionary Multi-Criterion Optimization (EMO-2015). Berlin, Germany: Springer-Verlag, 2015.

[26] K. Deb, S. Agrawal, A. Pratap, T. Meyarivan, A fast and elitist multiobjective genetic algorithm: NSGA-II, IEEE Transactions on Evolutionary Computation 6 (2) (2002) 182-197.

[27] A. Sinha, A. Srinivasan, K. Deb, A population-based, parent centric procedure for constrained real-parameter optimization, in: 2006 IEEE Congress on Evolutionary Computation (CEC-2006), IEEE Press, 2006, pp. 239-245.

[28] K. Deb, A. Sinha, An efficient and accurate solution methodology for bilevel multiobjective programming problems using a hybrid evolutionary-local-search algorithm, Evolutionary Computation Journal 18 (3) (2010) 403-449.

[29] E. Zitzler, L. Thiele, M. Laumanns, C. M. Fonseca, V. G. da Fonseca, Performance Assessment of Multiobjective Optimizers: An Analysis and Review, IEEE Transactions on Evolutionary Computation 7 (2) (2003) 117-132. 